\title{
Neutrino non-standard interactions and dark matter searches with multi-ton scale detectors
}

\author{
D. Aristizabal Sierra, ${ }^{a, b}$ N. Rojas ${ }^{a}$ and M.H.G. Tytgat ${ }^{c}$ \\ ${ }^{a}$ Universidad Técnica Federico Santa María - Departamento de Física, \\ Casilla 110-V, Avda. España 1680, Valparaíso, Chile \\ ${ }^{b}$ IFPA, Dép. AGO, Université de Liège, \\ Bât B5, Sart Tilman B-4000 Liège 1, Belgium \\ ${ }^{c}$ Université Libre de Bruxelles-Service de Physique Théorique CP225, \\ Blvd. du Triomphe (Campus de la Plaine), B-1050 Bruxelles, Belgium \\ E-mail: daristizabal@ulg.ac.be, nicolas.rojasro@usm.cl, \\ mtytgat@ulb.ac.be
}

ABSTRACT: Future dark matter (DM) direct detection searches will be subject to irreducible neutrino backgrounds that will challenge the identification of an actual WIMP signal in experiments without directionality sensitivity. We study the impact of neutrinoquark non-standard interactions (NSI) on this background, assuming the constraints from neutrino oscillations and the recent COHERENT experiment data, which are relevant for NSI mediated by light mediators, $m_{\text {med }} \lesssim \mathcal{O}(\mathrm{GeV})$. We calculate the expected number of neutrino-nucleus elastic scattering events in a Xe-based ton-size dark matter detector, including solar neutrino fluxes from the $p p$ chain and CNO cycle as well as sub-GeV atmospheric fluxes and taking into account NSI effects in both propagation and detection. We find that sizable deviations from the standard model expectation are possible, but are more pronounced for flavor-diagonal couplings, in particular for electron neutrinos. We show that neutrino NSI can enhance or deplete the neutrino-nucleus event rate, which may impact DM searches in multi-ton detectors.

Keywords: Neutrino Physics, Solar and Atmospheric Neutrinos

ArXiv EPrint: 1712.09667 


\section{Contents}

1 Introduction 1

2 Neutrino-quark non-standard interactions 3

3 Neutrino-nucleus elastic scattering events 5

3.1 Coherent neutrino-nucleus elastic cross section and NSI 5

$\begin{array}{ll}3.2 & \text { Solar neutrino flavor conversion and neutrino-nucleus scattering rates }\end{array}$

$\begin{array}{lll}3.3 & \text { Atmospheric neutrino-nucleus scattering rates } & 10\end{array}$

4 NSI at ton-size DM Xe detectors

$\begin{array}{lll}4.1 & \text { Implications for background-free sensitivities } & 15\end{array}$

$\begin{array}{lll}5 & \text { Conclusions } & 17\end{array}$

\section{Introduction}

A wealth of astrophysical and cosmological data support the idea that the dominant form of matter in the universe is made of dark matter (DM). Several DM candidates have been proposed ranging from astrophysical observable objects [1] up to fundamental particles [2]. Among the latter, the most studied candidates (both theoretically and experimentally) are massive particles whose abundance is determined by thermal freeze-out (weakly interacting massive particles or WIMPs).

WIMPs are being searched for using direct detection experiments since the late 80's, following a proposal by Goodman and Witten [3]. These experiments rely on energy deposition measurements from WIMP-induced nuclear recoil processes, which are expected to result as the Earth (detector) moves in the galactic DM halo. The non-observation of such signatures are translated into upper limits for the WIMP-nucleon (spin-independent or spin-dependent) cross section as a function of the DM mass. At present the most stringent bound is given by the XENON1T collaboration, which in the absence of a nuclear recoil signal has derived the limit $\sigma_{\chi-n} \lesssim 7.7 \times 10^{-47} \mathrm{~cm}^{2} / c^{2}$ for $m_{\chi}=35 \mathrm{GeV} / c^{2}$ at $90 \%$ CL [4]. Searches beyond these values and down to, say, $\sigma_{\chi-n} \sim 10^{-49} \mathrm{~cm}^{2} / c^{2}$ require multi-ton target masses, as planned for the DARWIN detector [5].

The reach of these experiments, however, will be limited by an irreducible neutrino background. Indeed, coherent neutrino nucleus elastic scattering $(\mathrm{C} \nu \mathrm{NES})$ induced by solar and, eventually, atmospheric neutrino fluxes will mimic a possible WIMP-nucleus signal [6]. While the precise extent of this background is affected by various factors, including neutrino flux uncertainties and astrophysical parameters $[6,7]$, in the SM, the C $\nu$ NES cross section is well understood and is not a source of uncertainty. This can potentially change in 
the presence of new physics. The most obvious way through which this can occur is via neutrino-quark non-standard interactions (NSI), which historically were introduced by Wolfenstein as a possible way to account for the solar neutrino deficit [8].

Neutrino NSI are constrained by several observations, including neutrino oscillation data [9], neutrino deep inelastic scattering experimental data (CHARM [10] and $\mathrm{NuTeV}[11]$ ) and, potentially, the measurement of the $\mathrm{C} \nu \mathrm{NES}$ process itself, recently observed by the COHERENT experiment [12] (see [13] for a review and [14] for the detailed analysis). CHARM and NuTeV set strong constraints on $\nu_{\mu}$-related NSI parameters, but their relevance for constraining $\mathrm{C} \nu \mathrm{NES}$ depends on the range of application of the NSI effective operators. In particular, these constraints do not apply if the NSI are mediated by a particle with mass $m_{\text {med }}<\mathcal{O}(1) \mathrm{GeV}$ [15]. In what follows we will consider the possibility that NSI are mediated by a particle in the mass range $10 \mathrm{MeV} \lesssim m_{\text {med }} \lesssim 1 \mathrm{GeV}$, range in which CHARM and NuTeV bounds do not apply, but one is still allowed to use the constraints on NSI as extracted from a combined analysis of COHERENT and oscillation data in ref. [14]. It is worth pointing out that in this mass range bounds are less severe for all flavor-diagonal NSI couplings, and so compared to the case where deep inelastic scattering data apply one expects larger deviations on the neutrino-nucleus event rates.

Specifically, using current constraints on neutrino NSI derived from neutrino oscillation and COHERENT data [14], in this paper we quantify the deviations on the C $\nu$ NES expected number of events in a Xe-based DM detector using an exposure $\mathcal{E}=1$ (ton-year). For that aim we consider solar neutrino fluxes for which we include neutrinos from the $p p$ chain $\left(p p\right.$, hep $\left.,{ }^{8} \mathrm{~B},{ }^{7} \mathrm{Be}, p e p\right)$ and $\mathrm{CNO}$ cycle $\left({ }^{13} \mathrm{~N},{ }^{15} \mathrm{O},{ }^{17} \mathrm{~F}\right)$. We consider as well sub-GeV atmospheric neutrino fluxes. For the solar sector analysis we calculate NSI propagation effects in the mass dominance limit $\left(\Delta m_{31}^{2} \rightarrow \infty\right)$ and for the calculation of the electron neutrino survival probability we take into account the distribution of neutrino production in the Sun according to the standard solar model (SSM) BS05 [16]. We quantify matter-induced effects in the atmospheric sector and find they are negligible $\left(H_{\mathrm{mat}} / H_{\mathrm{vac}} \lesssim 0.1\right)[17]$, however we include neutrino flavor oscillations in vacuum in the calculation of the number of neutrino-nucleus events.

The study of beyond-the-standard model physics in neutrino-quark interactions in the context of multi-ton DM detectors, has been recently the subject of various analyses. Ref. [18] studied the capability of such detectors to constraint light mediators scenarios, while ref. [19] instead considered scenarios where the new physics couples both neutrinos and DM. Ref. [20] considered the implications of neutrino NSI on neutrino backgrounds. The analysis was done for solar neutrinos and in particular for the ${ }^{8} \mathrm{~B}$ neutrino flux, for which solar neutrino fluxes can mimic a WIMP-nucleus scattering event. We extend upon this study by reanalyzing NSI effects in the case of lighter mediators, and so in the light of the recently released COHERENT data. We furthermore consider NSI effects all over solar neutrino energies and include sub-GeV atmospheric neutrino fluxes. The former is particularly relevant if one aims at setting e.g. background-free sensitivities for light WIMPS.

The rest of this paper is organized as follows. In section 2 we introduce neutrino NSI, first assuming they are generated by heavy mediators $\left(q^{2} \ll m_{X}^{2}\right)^{1}$ and review current

\footnotetext{
${ }^{1}$ They might as well proceed through the exchange of light mediators $\left(q^{2} \gg m_{X}^{2}\right)$. The results for this case will be presented elsewhere [21].
} 
experimental allowed ranges. In section 3 we discuss $\mathrm{C} \nu \mathrm{NES}$ in the presence of NSI, calculate electron neutrino survival probabilities in the Sun for various NSI data sets and study atmospheric vacuum conversion probabilities. In section 4 we present our main results, namely the maximum and minimum number of $\mathrm{C} \nu \mathrm{NES}$ events in ton-size DM detectors (Xe based). We perform a single-parameter analysis, c'est-à-dire we assume all NSI parameters are zero but one and vary that coupling within its experimental allowed range. Although constrained, this analysis enable us to capture the main features of what should be expected from neutrino NSI in near-future DM direct detection experiments. Finally, in section 5 we summarize and present our conclusions.

\section{Neutrino-quark non-standard interactions}

NSI can be parametrized in terms of the following effective Lagrangian (see e.g. [22]):

$$
\mathcal{L}_{\mathrm{Eff}}=-\sqrt{2} G_{F} \sum_{q=u, d} \overline{\boldsymbol{\nu}} \gamma_{\mu} P_{L}\left(\boldsymbol{\epsilon}^{\boldsymbol{q} \boldsymbol{V}}+\boldsymbol{\epsilon}^{\boldsymbol{q} \boldsymbol{A}} \gamma_{5}\right) \boldsymbol{\nu} \bar{q} \gamma^{\mu} q
$$

where $\boldsymbol{\nu}^{T}=\left(\nu_{e}, \nu_{\mu}, \nu_{\tau}\right)$ and $\boldsymbol{\epsilon}^{\boldsymbol{q} \boldsymbol{V}}$ and $\boldsymbol{\epsilon}^{\boldsymbol{q} \boldsymbol{A}}$ refer to $3 \times 3$ matrices in flavor space whose elements correspond to vector and vector-axial couplings. Hereafter we will denote flavorrelated matrices in bold-face. This parametrization assumes that the neutrino NSI are related to new physics at a scale $m_{\text {med }} \gg q$, with $q$ the characteristic energy momentum transferred in the relevant process. It can be as well that the new physics is related with a scale way below the typical momentum exchange (light mediator scenario), in which case a phenomenological analysis requires to take into account the dynamics of the mediator. Such models with light mediators have been discussed in, for instance [23-25]. Here, we focus on the intermediate possibility where CHARM and NuTeV constraints do not apply, while an effective approach is still valid so that the constraints on NSI parameters from the COHERENT data analyzed in [14] hold. In this regime, the constraints on the flavordiagonal NSI couplings are substantially weaker. For instance (see e.g. [13])

$$
\left|\epsilon_{\mu \mu}^{d V}\right|<0.042 \quad \text { (Atm. }+ \text { Acc.) } \longrightarrow-0.075<\epsilon_{\mu \mu}^{d V}<0.33 \quad \text { (Oscillation + COHERENT) }
$$

Accelerator constraints are irrelevant for mediator masses below $\mathcal{O}(1 \mathrm{GeV})$. The precise value is not important here, as long as it is larger than about $100 \mathrm{MeV}$, the maximal momentum exchange scale we will consider. Since [14] assumes $m_{\text {med }} \gtrsim 10 \mathrm{MeV}$, our analysis will be therefore limited to $10 \mathrm{MeV}<m_{\text {med }}<1 \mathrm{GeV}$. While a priori this seems a narrow window in parameter space, it is however for that window where the largest possible deviations - compared to SM expectations - on the neutrino-nucleus scattering rate are expected.

The interactions in (2.1) lead to forward coherent scattering (order $G_{F}$ interaction) and scattering (order $G_{F}^{2}$ interaction) processes such as $\mathrm{C} \nu \mathrm{NES}$. The former are responsible for matter potentials in the Sun and in the Earth and are entirely controlled by $\epsilon^{q \boldsymbol{V}}$. For the latter, the vector current determines spin-independent processes while the axial-vector current accounts for spin-dependent ones. Reactions rates driven by vector-axial currents are relatively smaller (in particular for large nuclei) and so we will not consider them. From now on then we drop the index $V$. 


\begin{tabular}{|l|c|c|c|}
\hline \multicolumn{4}{|c|}{ Oscillation data (LMA) } \\
\hline $\bar{\epsilon}_{e e}^{u}$ & {$[0.0,0.51]$} & $\bar{\epsilon}_{e e}^{d}$ & {$[0.02,0.51]$} \\
\hline $\bar{\epsilon}_{\tau \tau}^{u}$ & {$[-0.01,0.03]$} & $\bar{\epsilon}_{\tau \tau}^{d}$ & {$[-0.01,0.03]$} \\
\hline$\epsilon_{e \mu}^{u}$ & {$[-0.09,0.04]$} & $\epsilon_{e \mu}^{d}$ & {$[-0.09,0.04]$} \\
\hline$\epsilon_{e \tau}^{u}$ & {$[-0.14,0.14]$} & $\epsilon_{e \tau}^{d}$ & {$[-0.13,0.14]$} \\
\hline$\epsilon_{\mu \tau}^{u}$ & {$[-0.01,0.01]$} & $\epsilon_{\mu \tau}^{d}$ & {$[-0.01,0.01]$} \\
\hline
\end{tabular}

\begin{tabular}{|c|c|c|c|}
\hline \multicolumn{4}{|c|}{ Oscillation+COHERENT } \\
\hline$\epsilon_{e e}^{u}$ & {$[0.028,0.6]$} & $\epsilon_{e e}^{d}$ & {$[0.03,0.55]$} \\
\hline$\epsilon_{\mu \mu}^{u}$ & {$[-0.088,0.37]$} & $\epsilon_{\mu \mu}^{d}$ & {$[-0.075,0.33]$} \\
\hline$\epsilon_{\tau \tau}^{u}$ & {$[-0.09,0.38]$} & $\epsilon_{\tau \tau}^{d}$ & {$[-0.075,0.33]$} \\
\hline$\epsilon_{e \mu}^{u}$ & {$[-0.073,0.044]$} & $\epsilon_{e \mu}^{d}$ & {$[-0.07,0.04]$} \\
\hline$\epsilon_{e \tau}^{u}$ & {$[-0.15,0.13]$} & $\epsilon_{e \tau}^{d}$ & {$[-0.13,0.12]$} \\
\hline$\epsilon_{\mu \tau}^{u}$ & {$[-0.01,0.009]$} & $\epsilon_{\mu \tau}^{d}$ & {$[-0.009,0.008]$} \\
\hline
\end{tabular}

Table 1. 90\% CL allowed ranges for vector NSI couplings for up and down quarks. Ranges in the table to the left are derived from neutrino oscillation data only for the large mixing angle (LMA) case [9], while those in the table to the right arise from the combined analysis of oscillation and COHERENT data [14], and are the ones we use in our analysis. In the oscillation data table $\bar{\epsilon}_{i i}^{q}$ refers to $\epsilon_{i i}^{q}-\epsilon_{\mu \mu}^{q}(i=e, \tau)$.

Constraints on NSI are derived from neutrino oscillation $[9,13,26,27]$ and COHERENT data [12]. Let us discuss this in more detail. The presence of the vector current in (2.1) induce additional diagonal and non-diagonal matter potential terms which affect neutrino flavor evolution in the Sun and in the Earth (depending on the neutrino energy). They produce distortions in the neutrino oscillation probabilities that can be constrained by using solar, KamLAND, atmospheric and long-baseline neutrino data [9]. Limits on all flavor non-diagonal NSI couplings and two combinations of diagonal couplings e.g. $\epsilon_{i i}^{q}-\epsilon_{\mu \mu}^{q}$ $(i=e, \tau)$ can be derived this way. ${ }^{2}$ For comparison we show the $90 \%$ CL limits reported in [9] for the large mixing angle (LMA) case $^{3}$ in table 1.

The vector current modifies as well the C $\nu$ NES cross section, and so data from the recent measurement of the $\mathrm{C} \nu \mathrm{NES}$ process by the COHERENT experiment [12] can be used to set bounds on the vector NSI parameters. The low-energy neutrinos $\left(E_{\nu} \lesssim 50 \mathrm{MeV}\right)$ used by COHERENT result from accelerator-driven protons that strike a fixed mercury target thus producing pions. Their decay produce a monochromatic muon neutrino flux and muons that afterward decay, thus producing anti-muon and electron continuous neutrino fluxes. These data therefore enable placing bounds on $\epsilon_{e i}^{q}$ and $\epsilon_{\mu i}^{q}(i=e, \mu, \tau)[12,28]$. Overall, bounds can be placed by two independent experimental sources (order $G_{F}$ and order $G_{F}^{2}$ processes), but can be as well derived by combining both, resulting in more competitive limits. Such an analysis has been done in ref. [14], and these are the limits we use in the study of the impact of neutrino NSI in near-future DM detectors in section 2. For reference they are displayed in table 1 (table to the right).

\footnotetext{
${ }^{2}$ Since the evolution of the neutrino flavor eigenstates is invariant under a constant shift of the Hamiltonian $H \rightarrow H-\mathcal{C} \mathbb{I}$ (with $\mathcal{C}$ an arbitrary constant and $\mathbb{I}$ the identity matrix), neutrino oscillation experiments can only constraint diagonal elements differences.

${ }^{3}$ The combined analysis of oscillation and COHERENT data disfavor the so-called LMA-dark solution at the $3.1 \sigma(3.6 \sigma)$ level for up (down) quarks [14].
} 


\section{$3 \quad$ Neutrino-nucleus elastic scattering events}

\subsection{Coherent neutrino-nucleus elastic cross section and NSI}

$\mathrm{C} \nu \mathrm{NES}$ is a neutral-current process in which the momentum exchange is of order $q \lesssim$ $100 \mathrm{MeV}$. In the SM it is therefore well-described by the effective Lagrangian

$$
\mathcal{L}_{\mathrm{Eff}}^{\mathrm{SM}}=-\sqrt{2} G_{F} \sum_{q=u, d}\left(\bar{\nu}_{i} \gamma_{\mu} P_{L} \nu_{i}\right) \bar{q} \gamma^{\mu}\left(g_{V}^{q}+g_{A}^{q} \gamma_{5}\right) q
$$

where $g_{V}^{q}$ and $g_{A}^{q}$ are the SM vector and axial-vector couplings. The cross section for $\mathrm{C} \nu \mathrm{NES}$ with spinless nuclei is given by $[29,30]$

$$
\frac{d \sigma\left(E_{\nu}, E_{r}\right)}{d E_{r}}=\frac{G_{F}^{2}}{4 \pi} Q_{\mathrm{SM}}^{2} m_{N}\left(1-\frac{E_{r}}{E_{r}^{\max }\left(E_{\nu}\right)}\right) F^{2}\left(E_{r}\right) .
$$

Here $m_{N}$ is the nucleus mass and $E_{r}$ the nuclear recoil energy, whose maximum value, $E_{r}^{\max }$, is given by

$$
E_{r}^{\max }=\frac{2 E_{\nu}^{2}}{m_{N}+2 E_{\nu}} .
$$

Note that since $E_{\nu} \ll m_{N}\left(E_{\nu} \lesssim 100 \mathrm{MeV}\right.$ for the neutrino-nucleus scattering process to be coherent), $E_{r} / E_{r}^{\max } \simeq E_{r} m_{N} / 2 E_{\nu}^{2}$. The atomic number and number of neutrons are encoded in $Q_{\mathrm{SM}}=N-\left(1-4 \sin ^{2} \theta_{W}\right) Z$, from which one can see the $N^{2}$ enhancement of the $\mathrm{C} \nu \mathrm{NES}$ cross section. Finally, we use the Helm [31] form factor

$$
F^{2}\left(q r_{n}\right)=3 \frac{j_{1}\left(q r_{n}\right)}{q r_{n}} e^{-q^{2} s^{2} / 2}
$$

where $q$ can be traded to recoil energy through $q=6.92 \times 10^{-3} \sqrt{A E_{r}} \mathrm{fm}^{-1}, j_{1}(x)$ is the order-one spherical Bessel function of the first kind and for the different parameters in (3.4) we adopt the conventions from [32]. Nuclear radius according to

$$
r_{n}^{2}=c^{2}+\frac{7}{3} \pi^{2} a^{2}-5 s^{2},
$$

with skin thickness given by $s=0.9 \mathrm{fm}, a=0.52 \mathrm{fm}$ and $c=\left(1.23 A^{1 / 3}-0.6\right) \mathrm{fm}$.

$\mathrm{C} \nu \mathrm{NES}$ can take place with non-zero spin nuclei as well. For example, of the six most abundant Xe isotopes $\left({ }^{129} \mathrm{Xe},{ }^{130} \mathrm{Xe},{ }^{131} \mathrm{Xe},{ }^{132} \mathrm{Xe},{ }^{134} \mathrm{Xe},{ }^{136} \mathrm{Xe}\right){ }^{129} \mathrm{Xe}$ is a $J=1 / 2$ fermion state whereas ${ }^{131} \mathrm{Xe}$ is a $J=3 / 2$. To properly account for scattering with non-zero spin nuclei one has to include contributions from the axial-vector current in (3.1). These contributions, being related with the spin operator, are suppressed compared with those from the vector current. Thus, to a fairly good approximation, the $\mathrm{C} \nu \mathrm{NES}$ is well-described by (3.2) regardless of the spin of the target nuclei.

Once NSI are introduced, the $\mathrm{C} \nu \mathrm{NES}$ cross section is no longer flavor diagonal and so different neutrino flavors have different cross sections. For $\nu_{i}-N$ it reads $[33,34]$ :

$$
\frac{d \sigma_{\nu_{i}}}{d E_{r}}=\frac{G_{F}^{2}}{4 \pi} Q_{\nu_{i}}^{2} m_{N}\left(1-\frac{E_{r}}{E_{r}^{\max }\left(E_{\nu}\right)}\right) F^{2}\left(E_{r}\right) .
$$


where the only difference with respect to (3.2) arises from $Q_{\mathrm{SM}} \rightarrow Q_{\nu_{i}}$, with

$$
Q_{\nu_{i}}^{2}=4\left[-\frac{Q_{\mathrm{SM}}}{2}+N\left(\epsilon_{i i}^{u}+2 \epsilon_{i i}^{d}\right)+Z\left(2 \epsilon_{i i}^{u}+\epsilon_{i i}^{d}\right)\right]^{2}+\sum_{j \neq i} 4\left[N\left(\epsilon_{i j}^{u}+2 \epsilon_{i j}^{d}\right)+Z\left(2 \epsilon_{i j}^{u}+\epsilon_{i j}^{d}\right)\right]^{2} .
$$

Note that for light mediators $\left(m_{X}^{2} \ll q^{2}\right)$ this is no longer true, and a different recoil energy dependence is expected. If this is the case, the $\nu-N$ and WIMP-nucleon recoil spectra might differ, thus alleviating the effects of the solar and atmospheric neutrino backgrounds [21].

\subsection{Solar neutrino flavor conversion and neutrino-nucleus scattering rates}

Solar neutrinos $\left(\nu_{e}\right)$ are produced in regions near the solar core $\left(r \lesssim 0.2 R_{\odot}\right)$, with the exact distribution of neutrino production determined by the SSM. Once produced, electron neutrinos are subject to flavor conversion, governed by the vacuum and matter Hamiltonians according to

$$
i \frac{d}{d r}|\boldsymbol{\nu}\rangle=\left[\frac{1}{2 E_{\nu}} \boldsymbol{U} \boldsymbol{H}_{\mathrm{vac}} \boldsymbol{U}^{\dagger}+\boldsymbol{H}_{\mathrm{mat}}\right]|\boldsymbol{\nu}\rangle .
$$

Here $|\boldsymbol{\nu}\rangle^{T}=\left|\nu_{e}, \nu_{\mu}, \nu_{\tau}\right\rangle^{T}$ refers to the neutrino flavor eigenstate basis, $r$ to the neutrino propagation path, $\boldsymbol{U}=\boldsymbol{U}\left(\theta_{23}\right) \boldsymbol{U}\left(\theta_{13}\right) \boldsymbol{U}\left(\theta_{12}\right)$ is the leptonic mixing matrix $\left(U\left(\theta_{i j}\right)\right.$ is a $3 \times 3$ rotation matrix $), \boldsymbol{H}_{\mathrm{vac}}=\operatorname{diag}\left(0, \Delta m_{21}^{2}, \Delta m_{31}^{2}\right)$ and in the absence of NSI $\boldsymbol{H}_{\text {mat }}=$ $\sqrt{2} G_{F} n_{e}(r) \operatorname{diag}(1,0,0)$, with $n_{e}(r)$ the solar electron number density. Neutrino NSI induce additional matter potentials which change the matter Hamiltonian and thus affect neutrino flavor evolution. In full generality the evolution equation in (3.8) becomes

$$
i \frac{d}{d r}|\boldsymbol{\nu}\rangle=\left[\frac{1}{2 E_{\nu}} \boldsymbol{U} \boldsymbol{H}_{\mathrm{vac}} \boldsymbol{U}^{\dagger}+\sqrt{2} G_{F} n_{e}(r) \sum_{f=e, u, d} \varepsilon^{f}\right]|\boldsymbol{\nu}\rangle,
$$

where the NSI coupling matrices $\varepsilon^{f}$ read

$$
\varepsilon^{f}=\left(\begin{array}{ccc}
1+\varepsilon_{e e}^{f} & \varepsilon_{e \mu}^{f} & \varepsilon_{e \tau}^{f} \\
\varepsilon_{e \mu}^{f} & \epsilon_{\mu \mu}^{f} & \varepsilon_{\mu \tau}^{f} \\
\varepsilon_{e \tau}^{f} & \varepsilon_{\mu \tau}^{f} & \varepsilon_{\tau \tau}^{f}
\end{array}\right)
$$

with $\varepsilon_{i j}^{f}(r)=Y_{f}(r) \epsilon_{i j}^{f}(f=e, u, d)$ and $Y_{f}(r)=n_{f}(r) / n_{e}(r)$. The up- and down-quark relative abundances can be written in terms of the neutron relative abundance according to

$$
Y_{u}=2+Y_{n} \quad \text { and } \quad Y_{d}=1+2 Y_{n},
$$

with the neutron number density in turn calculated from the ${ }^{4} \mathrm{He}$ and ${ }^{1} \mathrm{H}$ mass fractions (metallicity in the Sun amounts to less than $1.5 \%$ so it can be safely neglected), namely

$$
n_{n}(r) \simeq \frac{X\left({ }^{4} \mathrm{He}\right)}{2 X\left({ }^{1} \mathrm{H}\right)+X\left({ }^{4} \mathrm{He}\right)}
$$


Since we are interested in $\mathrm{C} \nu \mathrm{NES}$, from now on we set $\epsilon_{i j}^{e}=0$ and following refs. [9, 14] we do not consider simultaneous contributions from up- and down-quark couplings. For all the SSM related quantities we use those from the BS05 SSM [16].

To a fairly good approximation, flavor conversion probabilities can be calculated in the mass dominance limit, $\Delta m_{31}^{2} \rightarrow \infty$. In this limit, and with vanishing CP-violating phases, neutrino propagation takes place in the basis $|\tilde{\boldsymbol{\nu}}\rangle=\boldsymbol{U}_{\mathbf{1 3}}{ }^{T} \boldsymbol{U}_{\mathbf{2 3}}{ }^{T}|\boldsymbol{\nu}\rangle$, which neglecting terms proportional to $\sin \theta_{13}$ corresponds to the following neutrino eigenstates:

$$
\tilde{\nu}_{e} \simeq \nu_{e}, \quad \tilde{\nu}_{\mu}=\cos \theta_{23} \nu_{\mu}-\sin \theta_{23} \nu_{\tau}, \quad \tilde{\nu}_{\tau} \simeq \sin \theta_{23} \nu_{\mu}+\cos \theta_{23} \nu_{\tau} .
$$

In this basis, which we dub as propagation basis, $\tilde{\nu}_{e}-\tilde{\nu}_{\tau}$ and $\tilde{\nu}_{\mu}-\tilde{\nu}_{\tau}$ mixing is of order $\xi_{i j}=G_{F} n_{q}(r) \epsilon_{i j}^{q} /\left(\Delta m_{31}^{2} / E_{\nu}\right)$. Taking $n_{q} \simeq 10^{26} \mathrm{~cm}^{-3}$ (up-quark number density at about $r=0.1 R_{\odot}$ ), $E_{\nu} \lesssim 20 \mathrm{MeV}$ and $\Delta m_{31}^{2}=2.55 \times 10^{-3} \mathrm{eV}^{2}$ (BFPV for normal ordering) [35], one can see that even for order-one NSI couplings $\xi_{i j} \ll 1$. This means that in the mass dominance limit, $\tilde{\nu}_{\tau}$ is decoupled and so neutrino flavor evolution can be studied in the two-flavor approximation with electron neutrino flavor conversion determined by $1-\left\langle\mathcal{P}_{e e}\left(E_{\nu}\right)\right\rangle$. Thus, in what follows we discuss the calculation of the averaged survival probability $\left\langle\mathcal{P}_{e e}\left(E_{\nu}\right)\right\rangle$. First of all, due to the quark and electron densities $\mathcal{P}_{e e}$ depends not only on $E_{\nu}$ but also on the neutrino propagation path $r$. $\mathcal{P}_{e e}\left(E_{\nu}, r\right)$ can be written as [36]

$$
\mathcal{P}_{e e}\left(E_{\nu}, r\right)=\cos ^{4} \theta_{13} \mathcal{P}_{\text {eff }}\left(E_{\nu}, r\right)+\sin ^{4} \theta_{13}
$$

where the $r$ dependence is introduced by the effective probability given by $[37]^{4}$

$$
\mathcal{P}_{\text {eff }}\left(E_{\nu}, r\right)=\frac{1+\cos 2 \theta_{M}(r) \cos 2 \theta_{12}}{2} .
$$

The mixing angle in matter is calculated from the diagonalization of the $2 \times 2$ Hamiltonian

$$
\boldsymbol{H}=\frac{1}{4 E_{\nu}}\left(\begin{array}{cc}
-\Delta m_{21}^{2} \cos 2 \theta_{12}+A & \Delta m_{21}^{2} \sin 2 \theta_{12}+B \\
\Delta m_{21}^{2} \sin 2 \theta_{12}+B & \Delta m_{21}^{2} \cos 2 \theta_{12}-A
\end{array}\right),
$$

where

$$
A=4 \sqrt{2} E_{\nu} G_{F} n_{e}(r)\left[\frac{\cos ^{2} \theta_{13}}{2}-Y_{q}(r) \varepsilon_{D}\right], \quad B=4 \sqrt{2} E_{\nu} G_{F} n_{e}(r) Y_{q}(r) \varepsilon_{N} .
$$

Note that in the limit $\epsilon_{i j}^{q}=0$ and $\cos \theta_{13}=0, A$ reduces to the SM term and $B$ vanishes. The parameters $\varepsilon_{D}$ and $\varepsilon_{N}$ result from the rotation from the flavor to the propagation basis and read [9]:

$$
\begin{aligned}
\varepsilon_{D}= & -\frac{c_{13}^{2}}{2} \epsilon_{e e}^{q}+\frac{\left[c_{13}^{2}-\left(s_{23}^{2}-s_{13}^{2} c_{23}^{2}\right)\right]}{2} \epsilon_{\mu \mu}^{q}+\frac{\left(s_{23}^{2}-c_{23}^{2} s_{13}^{2}\right)}{2} \epsilon_{\tau \tau}^{q}+s_{13} c_{13} s_{23} \epsilon_{e \mu}^{q} \\
& +s_{13} c_{13} c_{23} \epsilon_{e \mu}^{q}-c_{23} s_{23} \epsilon_{\mu \tau}^{q}, \\
\varepsilon_{N}= & -s_{13} c_{23} s_{23} \epsilon_{\mu \mu}^{q}+s_{13} c_{23} s_{23} \epsilon_{\tau \tau}^{q}+c_{13} c_{23} \epsilon_{e \mu}^{q}-c_{13} s_{23} \epsilon_{e \tau}^{q}+s_{13}\left(s_{23}^{2}-c_{23}^{2}\right) \epsilon_{\mu \tau}^{q} .
\end{aligned}
$$

Here we have adopted the notation $\cos \theta_{i j}=c_{i j}$ and $\sin \theta_{i j}=s_{i j}$. From eq. (3.16) the

\footnotetext{
${ }^{4}$ We have checked that $\gamma^{-1} \ll 1$, which guarantees neutrino adiabatic propagation, and so have taken the level-crossing probability $P_{c} \rightarrow 0$.
} 

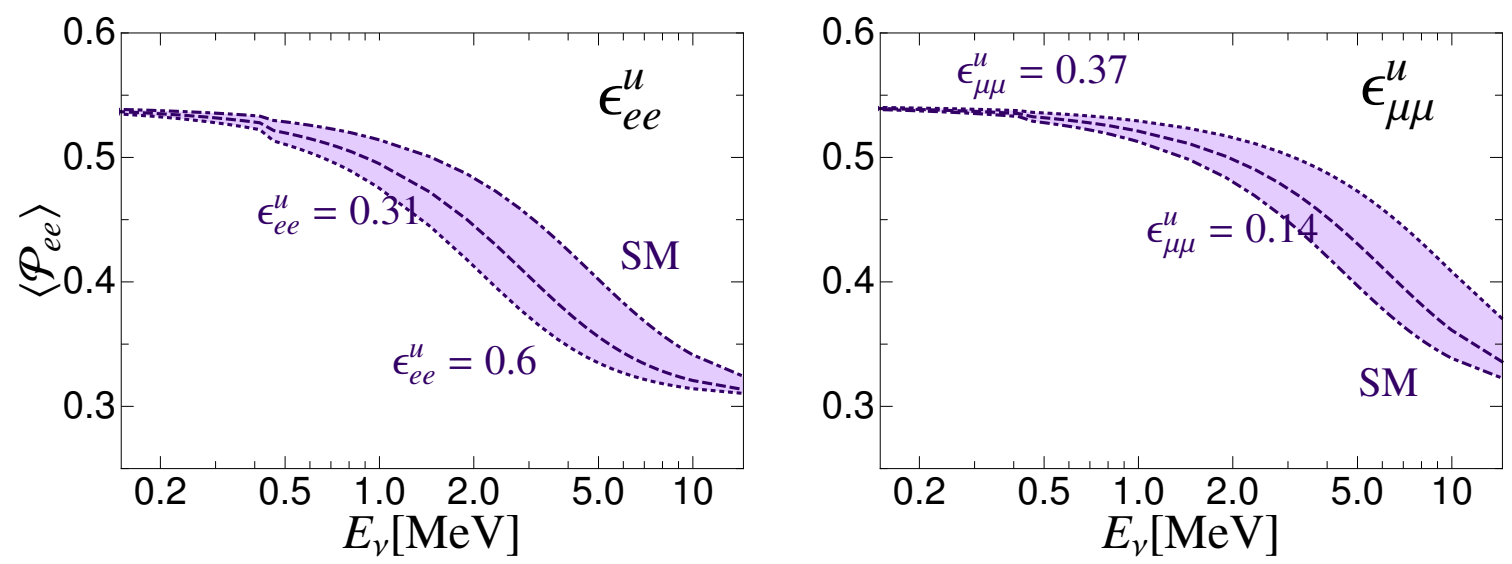

Figure 1. Averaged solar neutrino flavor oscillation probabilities for $\epsilon_{e e}^{u} \neq 0$ (left-hand side) and $\epsilon_{\mu \mu}^{u} \neq 0$ (right-hand side) as a function of the neutrino energy. Both NSI parameters have been varied in the ranges allowed by COHERENT and oscillation data [14] (see table 1, Oscillation+COHERENT). In both plots, the dotted-dashed curve refers to the SM case, the dotted curve to the amplitude obtained with the largest value for the corresponding coupling and the dashed for an intermediate value. The remaining NSI couplings in both cases have been put to zero.

mixing angle in matter can be straightforwardly calculated:

$$
\cos 2 \theta_{M}(r)=\frac{\Delta m_{12}^{2} \cos 2 \theta_{12}-A}{\sqrt{\left(\Delta m_{12}^{2} \cos 2 \theta_{12}-A\right)^{2}+\left(\Delta m_{12}^{2} \sin 2 \theta_{12}+B\right)^{2}}} .
$$

Eqs. (3.14) and (3.15) combined with eqs. (3.17)-(3.20) allow the determination of $\mathcal{P}_{e e}\left(E_{\nu}, r\right)$ in terms of neutrino oscillation parameters, electron and quark number densities and NSI parameters. The averaged survival probability is then obtained by integrating over $r$ taking into account the distribution of neutrino production in the Sun [9]:

$$
\left\langle\mathcal{P}_{e e}\left(E_{\nu}\right)\right\rangle=\frac{\sum_{\alpha} \Phi_{\alpha}\left(E_{\nu}\right) \int_{0}^{1} d r \rho_{\alpha}(r) \mathcal{P}_{e e}\left(E_{\nu}, r\right)}{\sum_{\alpha} \Phi_{\alpha}\left(E_{\nu}\right)}
$$

where $\Phi_{\alpha}\left(E_{\nu}\right)$ stands for neutrino fluxes and $\rho_{\alpha}(r)$ for the distribution of neutrino production, $\alpha=p p,{ }^{7} \mathrm{Be}$ (ground and excited state), pep, hep, ${ }^{8} \mathrm{~B},{ }^{13} \mathrm{~N},{ }^{15} \mathrm{O},{ }^{17} \mathrm{~F}$. In practice, to calculate $\left\langle\mathcal{P}_{e e}\left(E_{\nu}\right)\right\rangle$ we have followed a single-parameter analysis, varying only one NSI coupling at a time in the ranges allowed by the constraints derived from neutrino oscillation and COHERENT data [14]. These ranges have been split according to $\left.\epsilon_{i j}^{q}\right|_{n+1}=\left.\epsilon_{i j}^{q}\right|_{n}+\delta \epsilon_{i j}^{q}$ with $\delta \epsilon_{i j}^{q}=\left(\left.\epsilon_{i j}^{q}\right|_{\max }-\left.\epsilon_{i j}^{q}\right|_{\min }\right) / 10$. And the neutrino energy, $E_{\nu}$, has been varied from $0.145 \mathrm{MeV}$ to $14.5 \mathrm{MeV}$. The neutrino oscillation parameters have been fixed to their BFPVs according to ref. [35]. For each NSI parameter we thus have generated 11 data sets (11 averaged survival probabilities), including the SM case $\left(\epsilon_{i j}^{q}=0\right)$.

Focusing on up-quark NSI couplings (results for down-quark parameters resemble those of the up-quark case), the averaged survival probability deviates the most from the SM expectation for flavor-diagonal NSI and $\epsilon_{e \tau}^{u}$, being more pronounced for the former than 
for the latter. Figure 1 shows the result for $\left\langle\mathcal{P}_{e e}\right\rangle$ for the cases $\epsilon_{e e}^{u} \neq 0$ and $\epsilon_{\mu \mu}^{u} \neq 0$ (results for $\epsilon_{\tau \tau}^{u} \neq 0$ are pretty close to those found for $\epsilon_{\mu \mu}^{u} \neq 0$ ).

It can be seen that the range of variation of $\left\langle\mathcal{P}_{e e}\right\rangle$ has about the same amplitude in both cases. However its behavior with increasing values of the NSI couplings is rather different. For $\epsilon_{e e}^{u} \neq 0$, the averaged survival probability decreases with increasing values of $\epsilon_{e e}^{u}$ and its largest value (as a function of $E_{\nu}$ ) is indeed found when $\epsilon_{e e}=0$. For $\epsilon_{\mu \mu}^{u} \neq 0$, it is the other way around. Increasing values of the parameter leads to larger $\left\langle\mathcal{P}_{e e}\right\rangle$. This behavior can be readily understood as follows. In the SM case limit, $\epsilon_{i j}^{u}=0$, eq. (3.20) reduces to

$$
\cos 2 \theta_{M}=\frac{\Delta m_{21}^{2} \cos 2 \theta_{12}-2 \sqrt{2} E_{\nu} G_{F} n_{e} c_{13}^{2}}{\sqrt{\left(\Delta m_{21}^{2} \cos 2 \theta_{12}-2 \sqrt{2} E_{\nu} G_{F} n_{e} c_{13}^{2}\right)^{2}+\Delta m_{21}^{4} \sin ^{2} 2 \theta_{12}}} .
$$

The presence of the $\epsilon_{e e}^{u}$ and $\epsilon_{\mu \mu}^{u}$ couplings changes this equation by shifting either the first term in the denominator (numerator) or both. As can be seen from eqs. (3.17)-(3.20) the shift in the numerator of eq. (3.22) due to $\epsilon_{e e}^{u}$ is always negative. Thus, as $\epsilon_{e e}^{u}$ increases $\cos 2 \theta_{M}$ decreases and accordingly $\left\langle\mathcal{P}_{e e}\right\rangle$ decreases (see eqs. (3.14) and (3.15)). The shift due to $\epsilon_{\mu \mu}^{u}$ is instead positive thus leading to larger values of $\cos 2 \theta_{M}$ as $\epsilon_{\mu \mu}^{u}$ increases.

As we have pointed out, in the mass dominance limit solar neutrino flavor conversion can be reduced to a two-flavor oscillation problem, in which a mainly $\left|\nu_{e}\right\rangle$ state oscillates to a $\left|\tilde{\nu}_{\mu}\right\rangle$ state which is a superposition of $\left|\nu_{\mu}\right\rangle$ and $\left|\nu_{\tau}\right\rangle$ flavor eigenstates. Thus, in an Earth-based detector, neutrino-nucleus scattering will take place with either $\left|\nu_{e}\right\rangle$ or $\left|\tilde{\nu}_{\mu}\right\rangle$ and so the calculation of neutrino-nucleus scattering rates has to be done in the propagation basis as well. Rotating the effective Lagrangian in (2.1) to this basis, after dropping the axial-vector coupling matrix, results in

$$
\mathcal{L}_{\mathrm{Eff}}=-\sqrt{2} G_{F} \sum_{q=u, d} \overline{\tilde{\boldsymbol{\nu}}} \gamma_{\mu} P_{L} \tilde{\boldsymbol{\epsilon}} \tilde{\boldsymbol{q}} \tilde{\boldsymbol{\nu}} \bar{q} \gamma^{\mu} q
$$

with the NSI couplings in the propagation basis related with the NSI couplings in the flavor basis through the following transformation

$$
\widetilde{\boldsymbol{\epsilon}}=\boldsymbol{U}\left(\theta_{13}\right)^{T} \boldsymbol{U}\left(\theta_{23}\right)^{T} \boldsymbol{\epsilon}^{\boldsymbol{q}} \boldsymbol{U}\left(\theta_{23}\right) \boldsymbol{U}\left(\theta_{13}\right) .
$$

The differential cross sections for $\tilde{\nu}_{e}-N \simeq \nu_{e}-N$ and $\tilde{\nu}_{\mu}-N$ can then be obtained from (3.6) and (3.7) by $\epsilon_{i j}^{q} \rightarrow \tilde{\epsilon}_{i j}^{q}$. Results for the cases $\epsilon_{e e}^{u} \neq 0$ and $\epsilon_{\mu \mu}^{u} \neq 0$ are shown for both neutrino flavors in figure 2. As can be seen, deviations on either $d \sigma_{\nu_{e}} / d E_{r}$ or $d \sigma_{\tilde{\nu}_{\mu}} / d E_{r}$ are possible. The presence of NSI can enhance but also can deplete the flavored cross sections, thus potentially leading to substantial deviations of the expected neutrinonucleus scattering event rate.

Assuming a 100\% efficiency and with the oscillation probabilities and the $\nu-N$ differential cross sections calculated in the propagation basis, the $\nu-N$ event rate as a function of the recoil energy $E_{r}$ can be written according to [38]:

$$
\frac{d R_{\nu}^{\odot}}{d E_{r}}=N_{T} \sum_{\alpha} \int_{E_{\min }}^{\infty} \Phi_{\alpha}\left(E_{\nu}\right)\left[\left\langle\mathcal{P}_{e e}\right\rangle \frac{d \sigma_{\nu_{e}}}{d E_{r}}+\left(1-\left\langle\mathcal{P}_{e e}\right\rangle\right) \frac{d \sigma_{\tilde{\nu}_{\mu}}}{d E_{r}}\right] d E_{\nu} .
$$



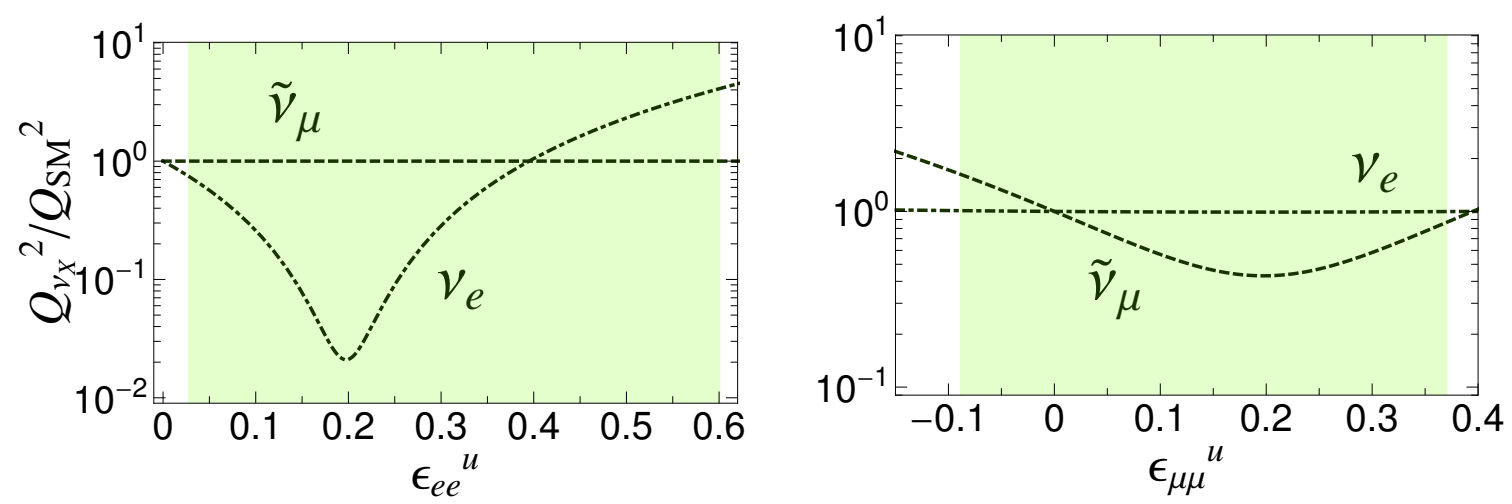

Figure 2. $\mathrm{C} \nu \mathrm{NES}$ cross sections normalized to the SM value as a function of the NSI parameters $\epsilon_{e e}^{u}$ (left-hand graph) and $\epsilon_{\mu \mu}^{u}$ (right-hand graph). Solar neutrino oscillations are analyzed in the mass dominance limit and so $\tilde{\nu}_{\mu} \simeq c_{23} \nu_{\mu}-s_{23} \nu_{\tau}$. The shaded regions indicate current allowed ranges for both parameters as required by neutrino oscillation and COHERENT data [14].

Here $N_{T}=N_{A} / A\left(N_{A}=6.022 \times 10^{29}\right.$ ton $\left.^{-1}\right), E_{r}^{\min }=\sqrt{m_{N} E_{r} / 2}$ and we include neutrinos emitted in the $p p$ and $\mathrm{CNO}$ chains, $\alpha=p p,{ }^{7} \mathrm{Be}$ (ground and excited state), pep, hep, ${ }^{8} \mathrm{~B}$, ${ }^{13} \mathrm{~N},{ }^{15} \mathrm{O},{ }^{17} \mathrm{~F}$, as in (3.21). The neutrino fluxes are in units of $\mathrm{cm}^{-2} \mathrm{year}^{-1} \mathrm{keV}^{-1}$, so the differential rates are in units of $\operatorname{ton}^{-1}$ year $^{-1} \mathrm{keV}^{-1}$ and the total event rates, $R_{\nu}^{\odot}$, are then measured in ton ${ }^{-1}$ year $^{-1}$. The latter are calculated from (3.25) by integration over $E_{r}$ from $E_{r}^{\text {th }}$ to $100 \mathrm{keV}$, with $E_{r}^{\text {th }} \subset\left[10^{-3}, 10^{2}\right] \mathrm{keV}$ and varied in steps $\delta E_{r}=\left(E_{r}^{n+1}-E_{r}^{n}\right) / 50$.

\subsection{Atmospheric neutrino-nucleus scattering rates}

In contrast to solar neutrinos, matter effects for sub-GeV atmospheric neutrinos are negligible and flavor conversion can be well described by vacuum oscillations [17]. This can be readily seen by considering the PREM model [39], for which $\left\langle n_{e}^{\oplus}\right\rangle=3.25 \times 10^{24} \mathrm{~cm}^{-3}$ and $\left\langle Y_{n}^{\oplus}\right\rangle=1.137$ both in the Earth core. With these values, using the BFPV for $\Delta m_{31}^{2}[35]$ and taking $E_{\nu}=100 \mathrm{MeV}$ so to minimize the vacuum term contribution one gets (see eq. (3.11))

$$
\frac{H_{\mathrm{mat}}}{H_{\mathrm{vac}}} \simeq 0.2 \times\left(\frac{2.55 \times 10^{-3}}{\Delta m_{31}^{2}}\right)\left(\frac{E_{\nu}}{100 \mathrm{MeV}}\right)\left(\frac{n_{e}^{\oplus}}{3.25 \times 10^{24} \mathrm{~cm}^{-3}}\right)\left(\frac{Y_{u}}{3.137}\right) \epsilon_{i j}^{u} .
$$

Note that this is the largest contribution from $H_{\text {mat }}$ to the neutrino flavor evolution equation, thus implying that matter effects for sub-GeV neutrinos can be fairly ignored.

Since only vacuum oscillations are relevant, the full three-flavor treatment is straightforward. In this case the oscillation probabilities can be further simplified by considering the limit $\Delta m_{21}^{2} \rightarrow 0$. They read [40]

$$
\mathcal{P}_{i j}\left(E_{\nu}, L\right)=4\left|U_{i 3}\right|^{2}\left|U_{j 3}\right|^{2} \sin ^{2}\left(\frac{\Delta m_{31}^{2}}{4 E_{\nu}} L\right),
$$

where $L$ refers to the distance covered by a neutrino from production at a height $h$ (perpendicular to the surface) to detection and is given by:

$$
L=\sqrt{\left(R_{\oplus}+h\right)^{2}-R_{\oplus}^{2} \sin ^{2} \theta_{z}}-R_{\oplus} \cos \theta_{z},
$$




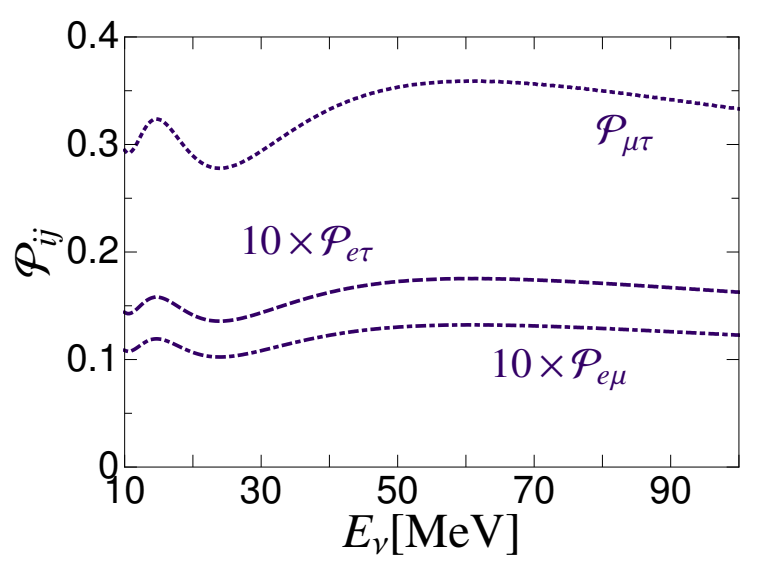

Figure 3. Atmospheric neutrino oscillations for sub-GeV neutrinos as a function of the neutrino energy $E_{\nu}$. For the calculation $\Delta m_{31}^{2}$ has been fixed to its BFPV according to ref. [35].

with $\theta_{z}$ the zenith angle of the neutrino trajectory. The oscillation probability in (3.27) is therefore a function of production height $h$ and zenith angle $\theta_{z}$. The distribution of vertical production height for sub-GeV neutrinos was calculated in [41] and its mean was found to be $\langle h\rangle \simeq 18 \mathrm{~km}$. Thus, rather than using the distribution we fix $h$ to this value and integrate over the zenith angle. The oscillation probabilities relevant for the determination of the $\nu-N$ event rate can then be written as

$$
\mathcal{P}_{i j}\left(E_{\nu}\right)=\frac{1}{\pi} \int_{-1}^{1} d \cos \theta \mathcal{P}_{i j}\left(E_{\nu}, L\right)
$$

As expected, neutrino flavor oscillations in this case are only relevant for $\nu_{\mu}-\nu_{\tau}$ conversion. The result is shown in figure 3 .

Since we set CP-violating phases to zero, the differential event rates associated with $\nu_{e}+\bar{\nu}_{e}$ and $\nu_{\mu}+\bar{\nu}_{\mu}$ fluxes can be written according to

$$
\begin{aligned}
& \frac{d R_{\nu_{e}+\bar{\nu}_{e}}^{\operatorname{Atm}}}{d E_{r}}=N_{T} \int_{E_{\min }}^{\infty} \Phi_{\nu_{e}+\bar{\nu}_{e}}\left(E_{\nu}\right)\left[\left(1-\mathcal{P}_{e \mu}-\mathcal{P}_{e \tau}\right) \frac{d \sigma_{\nu_{e}}}{d E_{r}}+\mathcal{P}_{e \mu} \frac{d \sigma_{\nu_{\mu}}}{d E_{r}}+\mathcal{P}_{e \tau} \frac{d \sigma_{\nu_{\tau}}}{d E_{r}}\right] \\
& \frac{d R_{\nu_{\mu}+\bar{\nu}_{\mu}}^{\mathrm{Atm}}}{d E_{r}}=N_{T} \int_{E_{\min }}^{\infty} \Phi_{\nu_{\mu}+\bar{\nu}_{\mu}}\left(E_{\nu}\right)\left[\mathcal{P}_{e \mu} \frac{d \sigma_{\nu_{e}}}{d E_{r}}+\left(1-\mathcal{P}_{e \mu}-\mathcal{P}_{\mu \tau}\right) \frac{d \sigma_{\nu_{\mu}}}{d E_{r}}+\mathcal{P}_{\mu \tau} \frac{d \sigma_{\nu_{\tau}}}{d E_{r}}\right]
\end{aligned}
$$

In (3.31) we assume time reversal invariance. We use the atmospheric neutrino fluxes from ref. [41], derived with the FLUKA Monte Carlo simulation package [42] and which provides fluxes for $\nu_{e}, \nu_{\mu}$ and the corresponding anti-neutrinos. The full differential $\nu-N$ event rate is obtained from (3.30) and (3.31) and the total event rate as in the solar case, integrating over $E_{r}$ from $E_{r}^{\text {th }}$ to $100 \mathrm{keV}$, with $E_{r}^{\text {th }} \subset\left[10^{-3}, 10^{2}\right] \mathrm{keV}$ and varied in steps $\delta E_{r}=\left(E_{r}^{n+1}-E_{r}^{n}\right) / 50$. The flavored cross sections are then given by (3.6) for which one finds sizable deviations from the SM only for diagonal NSI couplings, as expected given the tight constraints on the non-diagonal, particularly $\epsilon_{e \mu}^{u}$ and $\epsilon_{\mu \tau}^{u}$. The result for diagonal couplings is shown in figure 4 , in which all couplings but one have been assumed to be zero. 


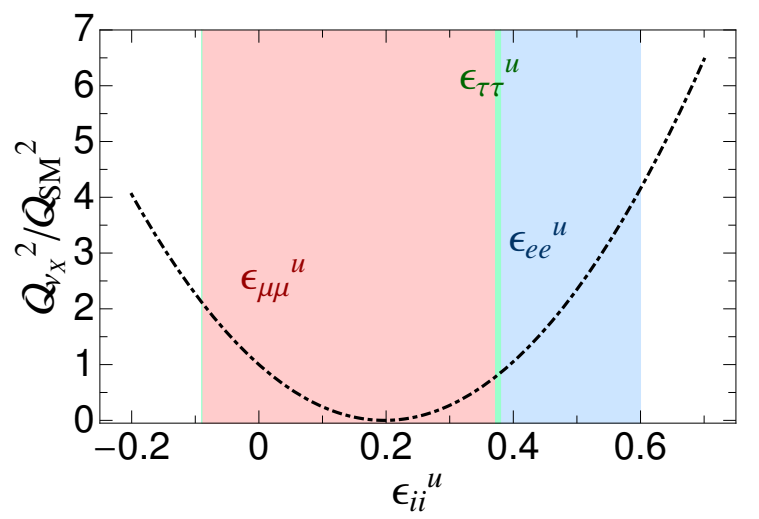

Figure 4. C $\nu$ NES cross section normalized to the SM value as a function of diagonal NSI couplings. Shaded regions refer to current limits on $\epsilon_{i i}^{u}(i=e, \mu, \tau)$ as required by neutrino oscillation and COHERENT data [14]. The blue shaded region $\left(\epsilon_{e e}^{u}\right)$ covers values down to 0.028 (see table 1). These are the cross sections which deviate the most from the SM value and that therefore lead to the largest deviations on the number of neutrino-nucleus scattering events in the atmospheric sector.

\section{NSI at ton-size DM Xe detectors}

We now turn to the discussion of the impact of neutrino NSI on the expected number of events in a Xe-based DM detector. For concreteness we assume an exposure of $\mathcal{E}=1$ ton $\times$ year. For practical reasons, and using the same approach we used in the calculation of the averaged survival probability in the Sun (see section 3.2), we perform a single-parameter analysis, considering only up-type couplings (results for down-type couplings resemble those found for the up-quark case). We first calculate the neutrino-nucleus differential event rate for ten equally-spaced values of the corresponding NSI parameter: $\left.\epsilon_{i j}^{u}\right|_{a+1}=\left.\epsilon_{i j}^{u}\right|_{a}+\delta \epsilon$, with $\delta \epsilon=\left(\left.\epsilon_{i j}^{u}\right|_{\max }-\left.\epsilon_{i j}^{u}\right|_{\min }\right) / 10$. From these results we then identify the parameters that maximize/minimize the recoil spectra, $d R_{\nu} / d E_{r}$. And for these parameters we calculate the neutrino-nucleus scattering events $\left(R_{\nu}\right)$ as a function of recoil energy threshold. ${ }^{5}$ For solar neutrinos fluxes we employ the results derived in section 3.2, eq. (3.25) combined with the averaged survival probabilities. For atmospheric neutrino fluxes we use the results from section 3.3. The results of our calculation are displayed in figure 5 , which we regard as the main result of this paper. Note that the vertical (horizontal) axes in that figure refer to $R_{\nu}$ (recoil energy threshold, $E_{r}$ ). Furthermore, we consider threshold energies as small as $10^{-3} \mathrm{keV}$ to show the effects that NSI have all over the solar neutrino spectrum, although such values are not attainable by a Xe-based detector any time soon.

One can see that even those parameters that are forced to be small by phenomenological constraints can have a sizable impact on the number of events. Departures from the SM value (horizontal line at 1 in figure 5) are energy dependent and are particularly pronounced in the solar-atmospheric transition, at $E_{r} \simeq 5.8 \mathrm{keV}$ when hep neutrinos reach their energy tail. Note that in the absence of NSI propagation effects, the SM and NSI neutrino-nucleus scattering rates differ only by a global numerical factor, $Q_{\mathrm{SM}} \rightarrow Q_{\nu_{i}}$ (see eq. (3.7)). Thus,

\footnotetext{
${ }^{5}$ We have cross checked that these parameters as well maximize/minimize $R_{\nu}$.
} 

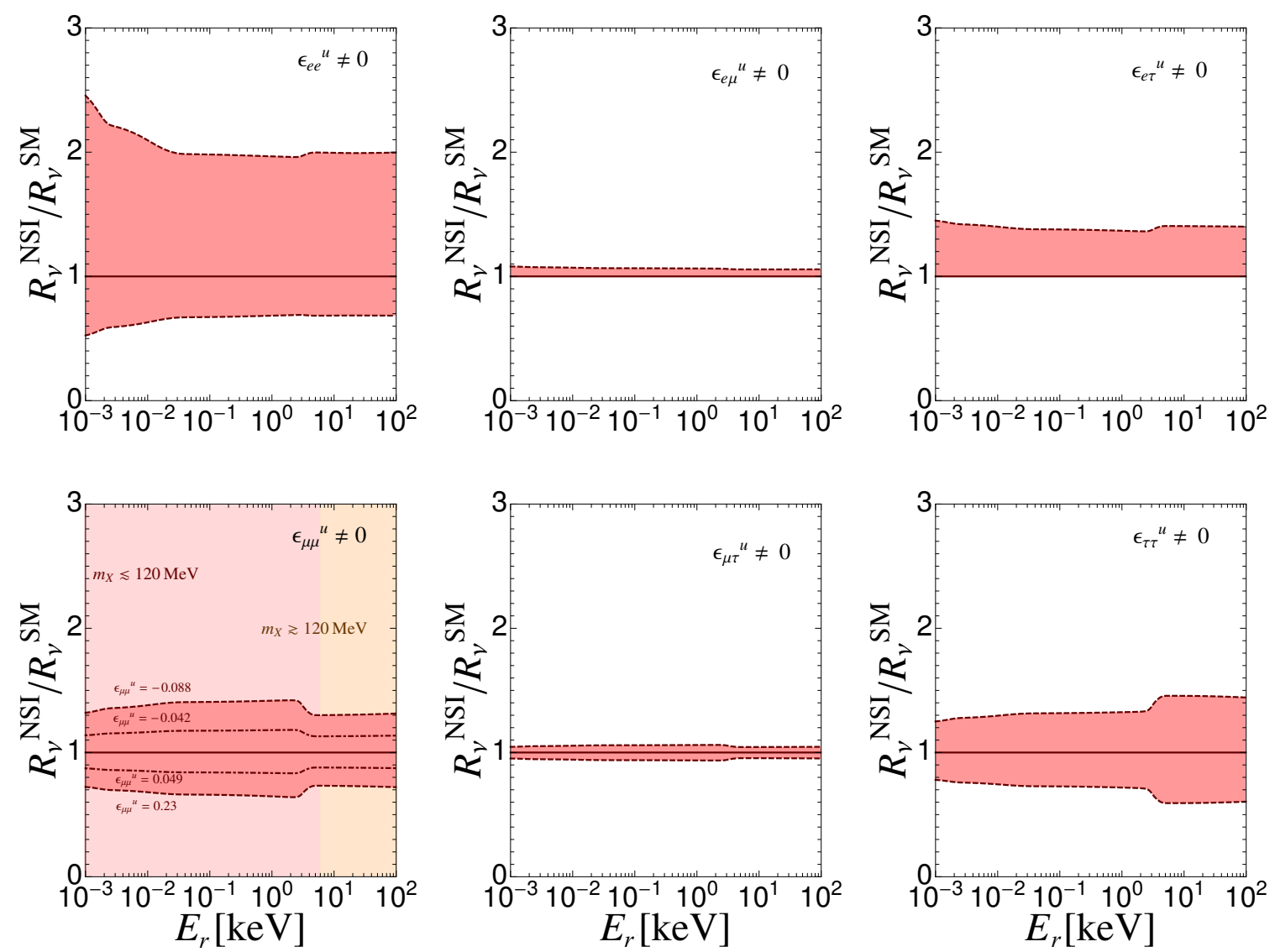

Figure 5. Expected number of neutrino-nucleus scattering events $R_{\nu}^{\mathrm{NSI}}$ normalized to the SM expectation $R_{\nu}^{\mathrm{SM}}$ as a function of recoil energy threshold and for a Xe-based DM detector assuming an exposure $\mathcal{E}=1$ ton $\times$ year and $100 \%$ efficiency. The results include $p p$, hep, ${ }^{7} \mathrm{Be}$ (ground and excited states), ${ }^{8} \mathrm{~B}$, pep, ${ }^{13} \mathrm{~N},{ }^{15} \mathrm{O},{ }^{17} \mathrm{~F}$ (CNO neutrinos) and atmospheric neutrinos fluxes (with $\left.E_{\nu}<100 \mathrm{MeV}\right)$.

this recoil energy dependence is a consequence of NSI propagation effects. Neutrino NSI not only lead to enhancements of the total number of events but can as well produce depletions (as firstly noted in ref. [20]), with both being relevant for DM direct detection experiments. The former places stronger limits on the sensitivity one can reach in a multiton DM detector, while the latter enables measurements of WIMP-nucleon cross sections that otherwise would be challenging reaching.

Overall, one observes that no matter the coupling a deviation from the SM expectation is always possible. However, diagonal couplings lead to the largest deviations which can either be enhancements or depletions of the event rate. Particularly important for solar neutrinos, are deviations in the energy range where the background is dominated by ${ }^{8} \mathrm{~B}$, since it is in that range where solar neutrinos can mimic a WIMP-nucleus signals [6]. In there, our findings show that $\epsilon_{\mu \mu}^{u}$ leads to enhancements of $R_{\nu}^{\mathrm{NSI}}$ which can exceed the SM value by about $40 \%$. For $\epsilon_{\tau \tau}^{u}$, enhancements are slightly smaller, but still substantial. For $\epsilon_{e e}^{u}$ they reach values of order 2 or so, being for this coupling the largest possible. This is 
somehow expected, as is for this coupling that bounds are less severe. Note that compared to what was found in ref. [20] this enhancement is larger, with the difference just due to the range employed. In ref. [20] $\left.\epsilon_{e e}^{u}\right|_{\max }=0.45$, value derived from oscillation and deep inelastic scattering data [15], while in our case $\left.\epsilon_{e e}^{u}\right|_{\max }=0.6$. This difference, although small, leads to a sufficiently large enhancement of the neutrino-nucleus cross section (see figure 2) that accounts for the larger deviation we found.

Since the largest deviations are found for diagonal NSI we focus in these cases, discussing in a bit more detail the results for $\epsilon_{e e}^{u}$ and $\epsilon_{\mu \mu}^{u}$. Maximization or minimization of $R_{\nu}$ is determined by the way in which the NSI parameters affect detection and propagation. The latter relevant only in the solar sector. For $\epsilon_{e e}^{u}$ we found $\left.R_{\nu}^{\mathrm{NSI}}\right|_{\max }$ at $\left.\epsilon_{e e}^{u}\right|_{\max }=0.6$ for both, the region where recoils are controlled by solar neutrinos fluxes $\left(E_{r} \lesssim 5.8 \mathrm{keV}\right)$ and the region governed by atmospheric neutrinos $\left(E_{r} \gtrsim 5.8 \mathrm{keV}\right)$. This can be understood as follows. In the solar region $d \sigma_{\tilde{\nu}_{\mu}} / d E_{r}$ does not depend on $\epsilon_{e e}^{u}$ and so it keeps always its SM value (see figure 2). The $\tilde{\nu}_{e}-N$ cross section instead reaches its maximum exactly at $\left.\epsilon_{e e}^{u}\right|_{\text {max }}$. The survival probability behaves in the opposite way, as $\epsilon_{e e}^{u}$ increases $\mathcal{P}_{e e}$ decreases globally, reaching minimum values exactly at $\left.\epsilon_{e e}^{u}\right|_{\max }$ (see figure 1, left graph). This effect tends to deplete the contribution of the $\tilde{\nu}_{e}-N$ scattering process to the total number of events. However, since for $\epsilon_{e e}^{u}=0.6$ the survival probability decreases at most $20 \%$ from its SM value, the overall effect is not sufficiently strong resulting in $\left.R_{\nu}^{\mathrm{NSI}}\right|_{\max }$ at $\left.\epsilon_{e e}^{u}\right|_{\max }$. $\left.R_{\nu}^{\mathrm{NSI}}\right|_{\min }$ is found at about the point where $d \sigma_{\tilde{\nu}_{e}} / d E_{r}$ reaches its minimum, $\epsilon_{e e}^{u} \simeq 0.2$. In the atmospheric sector, due $\mathcal{P}_{e \mu}, \mathcal{P}_{e \tau} \ll 1$ the event rate is mainly controlled by the $\nu_{e}-N$ cross section, which can be largely modified by NSI effects (see figures 3 and 4 ). This in turn is reflected in the non-depletion of $R_{\nu}^{\mathrm{NSI}}$ when passing from the solar to the atmospheric regions.

The parameter dependence in the case of $\epsilon_{\mu \mu}^{u}$ is rather different. For solar neutrinos, $\left.R_{\nu}^{\mathrm{NSI}}\right|_{\max }$ and $\left.R_{\nu}^{\mathrm{NSI}}\right|_{\min }$ are found where the $\tilde{\nu}_{\mu}-N$ cross section is maximized/minimized (see figure 2, right graph). When solar fluxes are no longer relevant and atmospheric kick in, the maximum expected number of events decreases. From (3.30) one can see that contributions from $\nu_{e}+\bar{\nu}_{e}$ fluxes are mainly SM, sizable deviations are due only to $\nu_{\mu}+\bar{\nu}_{\mu}$ fluxes. They are however not as large as in the case of $\epsilon_{e e}^{u}$ due to the sizable value of $\mathcal{P}_{\mu \tau}$ which tends to deplete the value of the second term in eq. (3.31), thus leading to small $\left.R_{\nu}^{\mathrm{NSI}}\right|_{\max }$.

The parameter dependence in the case $\epsilon_{\tau \tau}^{u}$ is rather similar to the one found in the $\epsilon_{\mu \mu}^{u}$ scenario in the solar sector, but in the atmospheric region the behavior is different. From eqs. (3.30) and (3.31) this is somehow expected. Contributions from electron neutrino fluxes barely deviate from SM values, substantial deviations can only arise from muon neutrino fluxes, with the $\nu_{\tau}-N$ cross section weighted by the $\nu_{u}-\nu_{\tau}$ oscillation probability which is of order 0.35 (see figure 4). This results in slightly larger deviations that those found in the $\epsilon_{\mu \mu}^{u}$ case. Non-diagonal NSI couplings are subject to tighter constraints, thus resulting in smaller effects. The exception being the case of the $\epsilon_{e \tau}^{u}$ parameter, for which we found no depletion and enhancements of order $40 \%$, inline with what was reported in [20].

The exact value (range) of the mediator mass for which our analysis is valid depends of course - on the recoil energy and hence on the neutrino sector, solar or atmospheric. For the $\epsilon_{\mu \mu}^{u} \neq 0$ case (figure 5 left top panel), we show the minimum mass that the mediator should have. In the solar sector masses below $\sim 120 \mathrm{MeV}$ suffice, with the exact value 
depending on the specific recoil energy. For recoil energies associated with atmospheric neutrinos fluxes, $m_{\text {med }} \gtrsim 120 \mathrm{MeV}$. Thus, while for solar neutrinos most of the mediator mass range is available, for atmospheric neutrinos the analysis is limited to a narrow window. However, as we have already pointed out is for this window where NSI effects may be expected to be maximized.

\subsection{Implications for background-free sensitivities}

$\mathrm{C} \nu \mathrm{NES}$ leads to recoil energy spectra which are closely degenerate with that induced by WIMP-nucleus scattering. As a result, once a direct detection experiment becomes sensitive to the neutrino background there is a limit on the WIMP cross section $\left(\sigma_{\mathrm{DM}-n}\right)$ that such experiment can explore, due to the inability to distinguish a neutrino- from a WIMPinduced signal. This limit defines the so-called neutrino "floor".

With the C $\nu$ NES process enhanced (diminished) by the neutrino-nucleus NSI, one expects the neutrino floor to be more (less) severe. Thus, in order to quantify the impact of NSI on the reach of next-generation DM detectors, we have quantified the 90\% CL sensitivities achievable. To do so, we have followed the method introduced in ref. [6] which enables the determination of the smallest WIMP cross section that can be measured for any WIMP mass in the presence of one-neutrino background event. It is worth pointing out that the limit on the WIMP cross section derived this way is just an estimate of what one should really expect. The precise determination of the saturation point, where the WIMP cross section one can access does not reduce with increasing (feasible) exposure, requires abandoning the background-free paradigm and using instead a likelihood analysis [6]. Here, however, we aim just at a preliminary understanding of the possible impact that NSI might have on direct detection sensitivities, and therefore stick to a simple background-free analysis.

We calculate the WIMP cross section as a function of the WIMP mass by fixing the WIMP-nucleus scattering event rate, $R_{\mathrm{DM}}$, to 2.3 (90\% CL sensitivity limits), namely

$$
\sigma_{\mathrm{DM}-n}=\frac{2.3}{\mathcal{E}\left(E_{r}^{\mathrm{th}}\right) \int_{E_{r}^{\mathrm{th}}}^{E_{\mathrm{max}}^{\max }} d R_{\mathrm{DM}} /\left.d E_{r}\right|_{\sigma_{\mathrm{DM}-n}=1}} .
$$

Here the WIMP event rate, which depends upon astrophysical, nuclear physics and particle physics parameters [32] reads

$$
\frac{d R_{\mathrm{DM}}}{d E_{r}}=\mathcal{E} \frac{\rho_{\mathrm{DM}} \sigma_{\mathrm{DM}-N}}{2 m_{\mathrm{DM}} \mu_{N}^{2}} F^{2}\left(E_{r}\right) \frac{k_{0}}{k} \frac{1}{\left(\pi v_{0}^{2}\right)^{3 / 2}} \int_{v_{\min }}^{v_{\mathrm{esc}}} e^{-\left|\boldsymbol{v}+\boldsymbol{v}_{\mathrm{lab}}\right|^{2} / v_{0}^{2}} \frac{d^{3} \boldsymbol{v}}{|\boldsymbol{v}|},
$$

where $\mathcal{E}$ is the exposure, $\rho_{\mathrm{DM}}$ is the DM density in the local halo, $\sigma_{\mathrm{DM}-N}$ is the DMnucleus cross section, $\mu_{N}$ is the DM-nucleus reduced mass, $v_{0}$ is the most likely speed of the DM in the halo, $F^{2}\left(E_{r}\right)$ is the form factor given in (3.4) and $\boldsymbol{v}$ is the velocity of the DM particles. The velocity distribution is Maxwellian and truncated at the DM escape velocity: $\left|\boldsymbol{v}+\boldsymbol{v}_{\text {lab }}\right|=\boldsymbol{v}_{\text {esc }}$, with $\boldsymbol{v}_{\text {lab }}$ the laboratory (Earth) velocity relative to the DM local halo. The factor $k_{0} / k$ is a normalization factor obtained by integrating the Maxwellian $\mathrm{DM}$ velocity distribution from 0 to $v_{\text {esc }}$ and is given by

$$
\frac{k_{0}}{k}=\left[\operatorname{erf}\left(\frac{v_{\mathrm{esc}}}{v_{0}}\right)-\frac{2}{\sqrt{\pi}} \frac{v_{\mathrm{esc}}}{v_{0}} e^{-v_{\mathrm{esc}}^{2} / v_{0}^{2}}\right]^{-1} .
$$




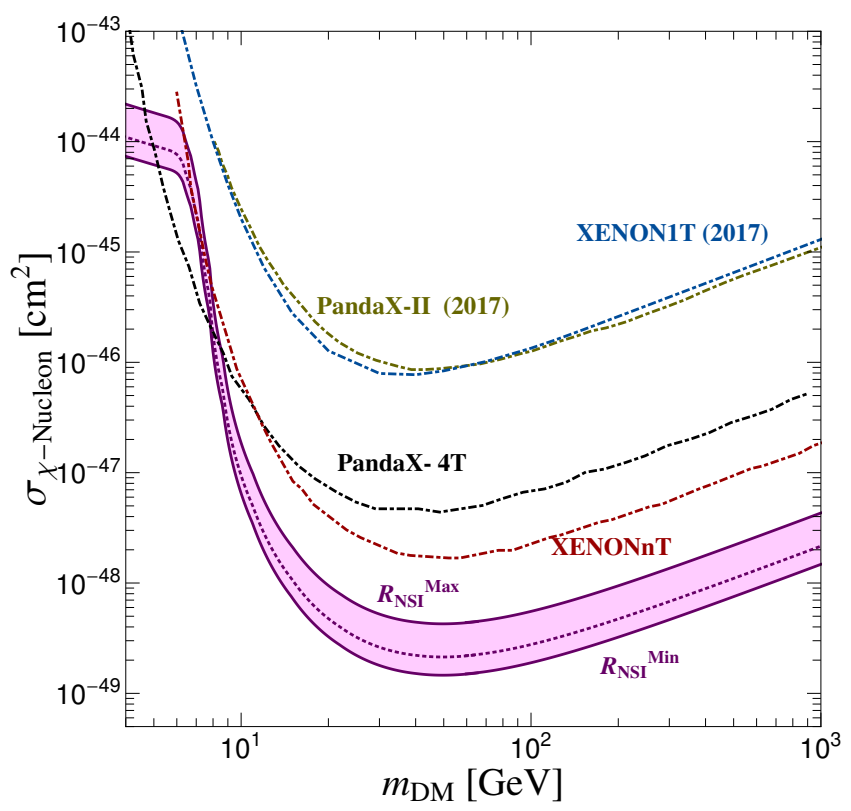

Figure 6. 90\% CL background-free sensitivities in the presence of neutrino-nucleus NSI. The purple shaded region indicates the effects of NSI on the background-free sensitivities. For comparison the SM 90\% CL background-free sensitivity curve is shown (dotted purple curve). These sensitivities have been derived by adjusting the exposure such that any measurement contains one-neutrino event [6]. It has been calculated for the case $\epsilon_{e e}^{u} \neq 0$.

Rather than integrating numerically the velocity distribution in (4.2) we have used its exact analytic form, namely [32]

$$
\int_{v_{\min }}^{v_{\text {esc }}} e^{-\left|\boldsymbol{v}+\boldsymbol{v}_{\mathrm{lab}}\right|^{2} / v_{0}^{2}} \frac{d^{3} \boldsymbol{v}}{|\boldsymbol{v}|}=2 \pi v_{0}^{2}\left\{\frac{\sqrt{\pi}}{4} \frac{v_{0}}{v_{\mathrm{lab}}}\left[\operatorname{erf}\left(\frac{v_{\text {min }}+v_{\mathrm{lab}}}{v_{0}}\right)-\operatorname{erf}\left(\frac{v_{\mathrm{min}}-v_{\mathrm{lab}}}{v_{0}}\right)\right]-e^{-v_{\mathrm{esc}}^{2} / v_{0}^{2}}\right\},
$$

with $v_{\text {min }}=\sqrt{2 E_{r} / m_{\mathrm{DM}} r} \times c$ and $r=4 m_{\mathrm{DM}} m_{N} /\left(m_{\mathrm{DM}}+m_{N}\right)^{2}$. We have written as well the DM-nucleus cross section in terms of the DM-nucleon cross section

$$
\sigma_{\mathrm{DM}-N}=\frac{\mu_{N}^{2}}{\mu_{n}^{2}} A^{2} \sigma_{\mathrm{DM}-n}
$$

with $\mu_{n}$ the DM-nucleon reduced mass. For the different astrophysical quantities we have used: $\rho_{\mathrm{DM}}=0.3 \mathrm{GeV} / \mathrm{c}^{2} / \mathrm{cm}^{3}, v_{0}=220 \mathrm{~km} / \mathrm{s}, v_{\text {lab }}=232 \mathrm{~km} / \mathrm{s}$ and $v_{\text {esc }}=544 \mathrm{~km} / \mathrm{s}$. For the integration we varied $E_{r}^{\text {th }}$ from $10^{-3}$ to $10^{2} \mathrm{keV}$ (with $10^{3}$ partitions) and took

$$
E_{r}^{\max }=\frac{m_{\mathrm{DM}}}{2}\left(\frac{v_{\mathrm{esc}}+v_{\mathrm{lab}}}{c}\right)^{2} \frac{r}{2}
$$

with $r$ given after eq. (4.4). The exposure has been tuned so to assure that each $E_{r}^{\text {th }}$ isocontour in the $\sigma_{\mathrm{DM}-n}-m_{\mathrm{DM}}$ plane contains one-neutrino event. This has been done by calculating the exposure as follows:

$$
\mathcal{E}\left(E_{r}^{\mathrm{th}}\right)=\frac{1 \text { neutrino event }}{\int_{E_{R}^{\mathrm{th}}} d R_{\nu} / d E_{r}} .
$$


The result is shown in figure 6, where we have plotted the SM sensitivities as well and included current (future) exclusion limits from XENON1T and PandaX-II [4, 43] (XENONnT and PandaX-4T [44]). The sensitivities shown in the plot refer to those obtained by calculating the exposure in (4.7) for the values of $\epsilon_{e e}^{u}$ that maximize/minimize $d R_{\nu} / d E_{r}$ (lower and upper curves in figure 5, left-top graph). The result in figure 6 show that the effects of neutrino-nucleus NSI, if present, work either way. They can worsen sensitivities or improve them. Note, that despite the effect being small it might have consequences. For example, if the NSI-induced depletion is effective (lower purple curve) the background-free sensitivities at XENONnT will be less affected. If instead the NSIinduced enhancement is present, XENONnT or PandaX-4T could start measuring neutrino scattering events sooner than expected.

\section{Conclusions}

The identification of a DM signal in next-to-next generation (multi-ton scale) detectors will be seriously challenged by irreducible solar and atmospheric neutrino backgrounds. Identifying all the features of this background is therefore mandatory to determine the implications it will have and the challenges it will pose. With this in mind, in this paper — following [20] — we have entertained the possibility that neutrinos are subject to NSI and have studied the impact they would have on the expected number of neutrino-nucleus scattering events in a Xe-based detector assuming an exposure of 1 ton · year. We have as well determine the background-free sensitivities achievable if these interactions are present, showing that they can either worsen or improve them, although only slightly in both cases.

Despite the recent observation of the C $\nu$ NES process by the COHERENT experiment and its consistency with the SM expectation, NSI couplings can still be relatively large, even when the COHERENT scattering data is combined with data from neutrino oscillation experiments. Thus, by using current constraints applicable in scenarios where neutrino NSI arise from mediators with masses in the range $\left[10,10^{3}\right] \mathrm{MeV}$, we have calculated the expected number of neutrino-nucleus scattering events in the presence of NSI including propagation and detection effects and extending the analysis over the solar and atmospheric neutrino sectors. In the former case, we have included all $p p$ and CNO solar neutrino fluxes, while in the latter case we have included $\nu_{e}, \nu_{\mu}, \bar{\nu}_{e}$ and $\bar{\nu}_{\mu}$ fluxes for neutrino energies up to $\sim 100 \mathrm{MeV}$. To simplify the computational burden, we have carried out a single-parameter analysis in which all couplings were put to zero but one and repeated the analysis for the remaining NSI couplings. Since we used constraints from [14], we analyzed up- and downquark couplings independently and presented only the results for the former, as the results for the latter are rather similar.

For the solar sector, we have studied propagation in the mass dominance limit and have taken into account the distribution of neutrino production within the Sun, for the calculation of the averaged neutrino survival probability. In the atmospheric region, Earth matter effects are negligible due to the largest relevant neutrino energies being below $\sim 100 \mathrm{MeV}$. In this sector then, NSI affect the expected number of neutrino-nucleus scattering events only 
through detection. We have anyway included vacuum oscillation probabilities (properly integrated over the zenith angle) in the determination of the number of scattering events.

Our main results are given by figure 5 and figure 6 and can be summarized as follows. Largest deviations from the SM expectation are more pronounced for flavor-diagonal NSI and can operate either enhancing the number of events or depleting them. For the most relevant recoil energy interval in the solar sector (that in which the neutrino flux is determined by ${ }^{8} \mathrm{~B}$ neutrinos in which the background can mimic WIMP-nucleon scattering events) the largest enhancement is found for $\epsilon_{e e}^{u}$ and can exceed the SM expectation up to a factor $\sim 2$. For $\epsilon_{\mu \mu}^{u}$ and $\epsilon_{\tau \tau}^{u}$, enhancements are smaller but still sizable. Depletions in that energy interval can amount to about $30-40 \%$ for all diagonal NSI parameters. Moving to higher neutrino energies we found that when atmospheric neutrino fluxes dominate the background, enhancements as large as twice the SM expectation are possible when $\epsilon_{e e}^{u}$ is present, in all other cases they are below this value but still are sizable. Depletions in the atmospheric sector can amount to about $50 \%$ if $\epsilon_{\tau \tau}^{u}$ is at work. For the off-diagonal couplings $\epsilon_{e \mu}^{u}$ and $\epsilon_{e \tau}^{u}$ we did not find any depletion, but we did for $\epsilon_{\mu \tau}^{u}$ although rather small (order 5\%) and so probably not experimentally measurable. Thus, if the neutrino-nucleus event rate for energies dominated by atmospheric neutrino fluxes is found to be below SM expectations - arguably - that will support the presence of new physics featuring flavordiagonal NSI, assuming one can disentangle the new interactions above any other effect.

Deviations, though small, might have an effect on the sensitivities achievable. To estimate the extent of such effects we have calculated the background-free sensitivities. We have shown that neutrino-nucleus NSI, if present, may worsen sensitivities or improve them. The effect is small, but might have observable consequences. We have stressed that if neutrino NSI are present either XENONnT or PandaX-4T, among others, will start observing neutrino-induced nuclear recoil events sooner than expected.

\section{Acknowledgments}

D.A.S. would like to thank Atri Bhattacharya, Jean-Rene Cudell, Thomas Hambye, Julian Heeck, Concha Gonzalez-Garcia, Florica Stancu and Mariam Tortola. In particular, Concha Gonzalez-Garcia for discussions regarding refs. [9, 15], Mariam Tortola for discussions on neutrino flavor oscillations in the Sun and Jean-Rene Cudell and Florica Stancu for useful conversations on nuclear physics. He would like also to thank the "Service de Physique Théorique" of the "Université Libre de Bruxelles" and the STAR research unit of the "Université de Liège" for the warm hospitality during the early stages of this work and partial financial support. This research has been supported by F.R.S.-FNRS through the program "Bourses de Séjour Scientifique" and by the Chilean grant "Unraveling new physics in the high-intensity and high-energy frontiers", Fondecyt No 1171136. NR was funded by proyecto FONDECYT Postdoctorado Nacional (2017) num. 3170135. The work of M.T. is supported by the FNRS-FRS, the Belgian Federal Science Policy Office through the Interuniversity Attraction Pole P7/37 and the Belgian Institute Interuniversitaire des Sciences Nucléaires (IISN). 
Open Access. This article is distributed under the terms of the Creative Commons Attribution License (CC-BY 4.0), which permits any use, distribution and reproduction in any medium, provided the original author(s) and source are credited.

\section{References}

[1] K. Griest, Galactic microlensing as a method of detecting massive compact halo objects, Astrophys. J. 366 (1991) 412 [INSPIRE].

[2] J.L. Feng, Dark matter candidates from particle physics and methods of detection, Ann. Rev. Astron. Astrophys. 48 (2010) 495 [arXiv:1003.0904] [INSPIRE].

[3] M.W. Goodman and E. Witten, Detectability of certain dark matter candidates, Phys. Rev. D 31 (1985) 3059 [INSPIRE].

[4] XENON collaboration, E. Aprile et al., First dark matter search results from the XENON1T experiment, Phys. Rev. Lett. 119 (2017) 181301 [arXiv:1705.06655] [INSPIRE].

[5] DARWIN collaboration, J. Aalbers et al., DARWIN: towards the ultimate dark matter detector, JCAP 11 (2016) 017 [arXiv:1606.07001] [INSPIRE].

[6] J. Billard, L. Strigari and E. Figueroa-Feliciano, Implication of neutrino backgrounds on the reach of next generation dark matter direct detection experiments, Phys. Rev. D 89 (2014) 023524 [arXiv: 1307.5458 ] [INSPIRE].

[7] C.A. O'Hare, Dark matter astrophysical uncertainties and the neutrino floor, Phys. Rev. D 94 (2016) 063527 [arXiv: 1604.03858] [INSPIRE].

[8] L. Wolfenstein, Neutrino oscillations in matter, Phys. Rev. D 17 (1978) 2369 [INSPIRE].

[9] M.C. Gonzalez-Garcia and M. Maltoni, Determination of matter potential from global analysis of neutrino oscillation data, JHEP 09 (2013) 152 [arXiv:1307.3092] [INSPIRE].

[10] CHARM collaboration, J. Dorenbosch et al., Experimental verification of the universality of $\nu_{e}$ and $\nu_{\mu}$ coupling to the neutral weak current, Phys. Lett. B 180 (1986) 303 [INSPIRE].

[11] NuTeV collaboration, G.P. Zeller et al., A precise determination of electroweak parameters in neutrino nucleon scattering, Phys. Rev. Lett. 88 (2002) 091802 [Erratum ibid. 90 (2003) 239902] [hep-ex/0110059] [INSPIRE].

[12] COHERENT collaboration, D. Akimov et al., Observation of coherent elastic neutrino-nucleus scattering, Science 357 (2017) 1123 [arXiv:1708.01294] [INSPIRE].

[13] Y. Farzan and M. Tortola, Neutrino oscillations and non-standard interactions, Front. in Phys. 6 (2018) 10 [arXiv:1710.09360] [INSPIRE].

[14] P. Coloma, M.C. Gonzalez-Garcia, M. Maltoni and T. Schwetz, COHERENT enlightenment of the neutrino dark side, Phys. Rev. D 96 (2017) 115007 [arXiv:1708.02899] [INSPIRE].

[15] P. Coloma, P.B. Denton, M.C. Gonzalez-Garcia, M. Maltoni and T. Schwetz, Curtailing the dark side in non-standard neutrino interactions, JHEP 04 (2017) 116 [arXiv:1701.04828] [INSPIRE].

[16] J.N. Bahcall, A.M. Serenelli and S. Basu, New solar opacities, abundances, helioseismology and neutrino fluxes, Astrophys. J. 621 (2005) L85 [astro-ph/0412440] [INSPIRE].

[17] A. Friedland, C. Lunardini and M. Maltoni, Atmospheric neutrinos as probes of neutrino-matter interactions, Phys. Rev. D 70 (2004) 111301 [hep-ph/0408264] [InSPIRE]. 
[18] D.G. Cerdeño, M. Fairbairn, T. Jubb, P.A.N. Machado, A.C. Vincent and C. Bœhm, Physics from solar neutrinos in dark matter direct detection experiments, JHEP 05 (2016) 118 [Erratum ibid. 09 (2016) 048] [arXiv: 1604.01025] [INSPIRE].

[19] E. Bertuzzo, F.F. Deppisch, S. Kulkarni, Y.F. Perez Gonzalez and R. Zukanovich Funchal, Dark matter and exotic neutrino interactions in direct detection searches, JHEP 04 (2017) 073 [Erratum ibid. 04 (2017) 073] [arXiv: 1701.07443] [INSPIRE].

[20] B. Dutta, S. Liao, L.E. Strigari and J.W. Walker, Non-standard interactions of solar neutrinos in dark matter experiments, Phys. Lett. B 773 (2017) 242 [arXiv:1705.00661] [INSPIRE].

[21] D. Aristizabal Sierra, N. Rojas and V.D. Romeri, Neutrino floor and non-standard interactions with light mediators, in preparation.

[22] T. Ohlsson, Status of non-standard neutrino interactions, Rept. Prog. Phys. 76 (2013) 044201 [arXiv: 1209.2710 ] [INSPIRE].

[23] Y. Farzan, A model for large non-standard interactions of neutrinos leading to the LMA-dark solution, Phys. Lett. B 748 (2015) 311 [arXiv:1505.06906] [INSPIRE].

[24] Y. Farzan and I.M. Shoemaker, Lepton flavor violating non-standard interactions via light mediators, JHEP 07 (2016) 033 [arXiv: 1512.09147] [INSPIRE].

[25] K.S. Babu, A. Friedland, P.A.N. Machado and I. Mocioiu, Flavor gauge models below the Fermi scale, JHEP 12 (2017) 096 [arXiv:1705.01822] [INSPIRE].

[26] A. Bolanos, O.G. Miranda, A. Palazzo, M.A. Tortola and J.W.F. Valle, Probing non-standard neutrino-electron interactions with solar and reactor neutrinos, Phys. Rev. D 79 (2009) 113012 [arXiv:0812.4417] [INSPIRE].

[27] F.J. Escrihuela, O.G. Miranda, M.A. Tortola and J.W.F. Valle, Constraining nonstandard neutrino-quark interactions with solar, reactor and accelerator data, Phys. Rev. D 80 (2009) 105009 [Erratum ibid. D 80 (2009) 129908] [arXiv: 0907.2630] [INSPIRE].

[28] J. Liao and D. Marfatia, COHERENT constraints on nonstandard neutrino interactions, Phys. Lett. B 775 (2017) 54 [arXiv:1708.04255] [INSPIRE].

[29] D.Z. Freedman, Coherent neutrino nucleus scattering as a probe of the weak neutral current, Phys. Rev. D 9 (1974) 1389 [INSPIRE].

[30] D.Z. Freedman, D.N. Schramm and D.L. Tubbs, The weak neutral current and its effects in stellar collapse, Ann. Rev. Nucl. Part. Sci. 27 (1977) 167 [inSPIRE].

[31] R.H. Helm, Inelastic and elastic scattering of $187 \mathrm{MeV}$ electrons from selected even-even nuclei, Phys. Rev. 104 (1956) 1466 [INSPIRE].

[32] J.D. Lewin and P.F. Smith, Review of mathematics, numerical factors and corrections for dark matter experiments based on elastic nuclear recoil, Astropart. Phys. 6 (1996) 87 [INSPIRE].

[33] J. Barranco, O.G. Miranda and T.I. Rashba, Probing new physics with coherent neutrino scattering off nuclei, JHEP 12 (2005) 021 [hep-ph/0508299] [INSPIRE].

[34] M. Lindner, W. Rodejohann and X.-J. Xu, Coherent neutrino-nucleus scattering and new neutrino interactions, JHEP 03 (2017) 097 [arXiv: 1612.04150] [INSPIRE].

[35] P.F. de Salas, D.V. Forero, C.A. Ternes, M. Tortola and J.W.F. Valle, Status of neutrino oscillations 2017, arXiv:1708.01186 [INSPIRE]. 
[36] T.-K. Kuo and J.T. Pantaleone, The solar neutrino problem and three neutrino oscillations, Phys. Rev. Lett. 57 (1986) 1805 [INSPIRE].

[37] S.J. Parke, Nonadiabatic level crossing in resonant neutrino oscillations, Phys. Rev. Lett. 57 (1986) 1275 [INSPIRE].

[38] T.-K. Kuo and J.T. Pantaleone, Neutrino oscillations in matter, Rev. Mod. Phys. 61 (1989) 937 [INSPIRE].

[39] A.M. Dziewonski and D.L. Anderson, Preliminary reference earth model, Phys. Earth Planet. Interiors 25 (1981) 297 [INSPIRE].

[40] E.K. Akhmedov, Neutrino physics, in Proceedings, Summer School in Particle Physics, Trieste Italy, 21 June-9 July 1999, pg. 103 [hep-ph/0001264] [INSPIRE].

[41] G. Battistoni, A. Ferrari, T. Montaruli and P.R. Sala, The atmospheric neutrino flux below $100 \mathrm{MeV}$ : the FLUKA results, Astropart. Phys. 23 (2005) 526 [INSPIRE].

[42] A. Ferrari, P.R. Sala, A. Fasso and J. Ranft, FLUKA: a multi-particle transport code (program version 2005), (2005) [INSPIRE].

[43] PandaX-II collaboration, X. Cui et al., Dark matter results from 54-ton-day exposure of PandaX-II experiment, Phys. Rev. Lett. 119 (2017) 181302 [arXiv:1708.06917] [InSPIRE].

[44] The PandaX-4T Dark Matter Experiment webpage, https://pandax.physics.sjtu.edu.cn. 